\title{
Time Course of Brain Network Reconfiguration Supporting Inhibitory Control
}

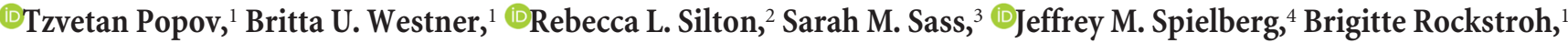 \\ Wendy Heller, ${ }^{5}$ and Gregory A. Miller ${ }^{5,6,7}$ \\ ${ }^{1}$ Department of Psychology, University of Konstanz, 78464 Konstanz, Germany, ${ }^{2}$ Department of Psychology, Loyola University, Chicago, Illinois 60660, \\ ${ }^{3}$ Department of Psychology, University of Texas, Tyler, Texas 75799, ${ }^{4}$ Department of Psychological and Brain Sciences, University of Delaware, Newark, \\ Delaware 19716, ${ }^{5}$ Department of Psychology, University of Illinois, Urbana-Champaign, Illinois 61820, ${ }^{6}$ Department of Psychology, and ${ }^{7}$ Department of \\ Psychiatry and Biobehavioral Sciences, University of California, Los Angeles, California 90095
}

Hemodynamic research has recently clarified key nodes and links in brain networks implementing inhibitory control. Although fMRI methods are optimized for identifying the structure of brain networks, the relatively slow temporal course of fMRI limits the ability to characterize network operation. The latter is crucial for developing a mechanistic understanding of how brain networks shift dynamically to support inhibitory control. To address this critical gap, we applied spectrally resolved Granger causality (GC) and random forest machine learning tools to human EEG data in two large samples of adults (test sample $n=96$, replication sample $n=237$, total $N=333$, both sexes) who performed a color-word Stroop task. Time-frequency analysis confirmed that recruitment of inhibitory control accompanied by slower behavioral responses was related to changes in theta and alpha/beta power. GC analyses revealed directionally asymmetric exchanges within frontal and between frontal and parietal brain areas: top-down influence of superior frontal gyrus (SFG) over both dorsal ACC (dACC) and inferior frontal gyrus (IFG), dACC control over middle frontal gyrus (MFG), and frontal-parietal exchanges (IFG, precuneus, MFG). Predictive analytics confirmed a combination of behavioral and brain-derived variables as the best set of predictors of inhibitory control demands, with SFG theta bearing higher classification importance than dACC theta and posterior beta tracking the onset of behavioral response. The present results provide mechanistic insight into the biological implementation of a psychological phenomenon: inhibitory control is implemented by dynamic routing processes during which the target response is upregulated via theta-mediated effective connectivity within key PFC nodes and via beta-mediated motor preparation.

Key words: EEG; Granger causality; inhibitory control; machine learning; neuronal oscillations; theta, alpha

\section{Significance Statement}

Hemodynamic neuroimaging research has recently clarified regional structures in brain networks supporting inhibitory control. However, due to inherent methodological constraints, much of this research has been unable to characterize the temporal dynamics of such networks (e.g., direction of information flow between nodes). Guided by fMRI research identifying the structure of brain networks supporting inhibitory control, results of EEG source analysis in a test sample $(n=96)$ and replication sample $(n=$ 237) using effective connectivity and predictive analytics strategies advance a model of inhibitory control by characterizing the precise temporal dynamics by which this network operates and exemplify an approach by which mechanistic models can be developed for other key psychological processes.

\section{Introduction}

Interference control during goal-directed behavior is a key cognitive operation. Many aspects of daily life require rapid yet ac-

\footnotetext{
Received Sept. 14, 2017; revised March 20, 2018; accepted March 26, 2018.

Author contributions: W.H. and G.A.M. designed research; R.L.S. and S.M.S. performed research; T.P. and B.U.W. analyzed data; T.P., B.U.W., R.L.S., S.M.S., J.M.S., B.R., W.H., and G.A.M. wrote the paper.

This work was supported by the National Institute of Mental Health-National Institutes of Health (Grants P50

MH079485, R01 MH61358, and R21 DA14111) and the Deutsche Forschungsgemeinschaft (Grant R0805/17).

The authors declare no competing financial interests.

Correspondence should be addressed to Tzvetan Popov, University of Konstanz, PF 905, 78464 Konstanz, Ger-

many. E-mail: tzvetan.popov@uni-konstanz.de.
}

curate control of an indefinite amount of information by finite processing resources. Two overlapping lines of research pursuing such mechanisms are studies of large-scale cerebral connectivity and of neuronal oscillations. Cognitive operations might be better understood by incorporating knowledge of the network of anatomical connections and of the periodicity of neuronal activity that structures information to identify mechanisms of processing and their disruption in psychopathology. However, parameteriza- 
tion and interpretation of such data are often difficult due to the enormously high degrees of freedom inherent in functional brain data.

In a recent hemodynamic neuroimaging study, our group used graph theory, a formal description of a network and its emergent properties, to identify a particular network constellation optimized for specialized processing during increased demands for inhibitory control (Spielberg et al., 2015). This network was characterized by increased connectivity orchestrated primarily by dorsal ACC (dACC) and inferior frontal regions showing a flexible organization predictive of behavioral outcome. Given the temporal limits of conventional fMRI, that study could not determine possible causal relationships between nodes within this network, such as whether dACC has top-down influence on inferior frontal regions in the network.

Two large samples were combined to address these unresolved issues using recent advances in EEG analysis complemented by machine-learning techniques. EEG data from 96 participants in the fMRI study performing the same task during dense-array EEG recording typically within 2 weeks of the fMRI session were combined with EEG data from a replication sample of 237 participants to develop a mechanistic account of internodal network communication. Initial analyses characterized the time-frequency profiles of network constituents and quantified their relationship to behavioral performance. Spectrally resolved Granger causality (GC) analyses quantified key parameters of a given relationship, its directionality and frequency. Distinct frequency bands are theorized to represent coordinated state changes across local networks, so the present study evaluated the role of theta $(4-8 \mathrm{~Hz})$ and alpha/beta $(10-30 \mathrm{~Hz})$ in networks associated with topdown control and behavioral performance (reaction time, RT). Finally, random forest classification analysis was used to identify the importance of neural activity per network node for predicting individual brain states characterized by high versus low demand for inhibitory control.

Evidence suggests that top-down control can be accomplished via oscillatory activity $<30 \mathrm{~Hz}$ (Cohen et al., 2012; van Kerkoerle et al., 2014; Bastos et al., 2015; Chmielewski et al., 2016; Michalareas et al., 2016; Wang et al., 2016; Babapoor-Farrokhran et al., 2017; Popov et al., 2017; Richter et al., 2017; Schoffelen et al., 2017). More specifically, theta activity likely reflects the coordination of a distributed network engaged in inhibitory control (Chmielewski et al., 2016; Babapoor-Farrokhran et al., 2017) and memory (Anderson et al., 2010) processes. Accordingly, we predicted that connectivity between PFC and dACC (per Spielberg et al., 2015) would be directed and frequency specific via theta activity.

Multiple frequency bands are expected to be implicated in a given task, modulating task-specific networks (Engel and Fries, 2010), and experimental and theoretical models have suggested upregulation of the strength of the desired response as a critical feature of inhibitory control (Munakata et al., 2011). Therefore, the network configuration was predicted to shift toward increased influence of PFC. Specifically, this upregulation would be reflected in modulation of alpha/beta power in motor regions. Beta-band activity has been theorized to signal maintenance of a sensorimotor set to facilitate efficient processing of feedback and recalibration of the sensorimotor system (Engel and Fries, 2010). Likely indicating a change in the status quo, decreased beta power in primary motor cortex has been observed when inhibitory control is exerted in stop signal tasks (Swann et al., 2009; Wheaton et al., 2009). Therefore, a decrease in network beta power was an- ticipated to be related to slower response time, particularly in the context of incongruent trials.

\section{Materials and Methods}

Participants. A total of 333 (208 female, age $26.25 \pm 10.28$ years) participants were recruited from the community. Exclusion criteria were as follows: left handedness determined by the Edinburgh Handedness Inventory (Oldfield, 1971), head trauma with loss of consciousness, psychoactive medication, abnormal color vision, claustrophobia, recent drug/alcohol use, excessive caffeine intake, and lack of sleep.

Experimental design. Experimental procedures were those reported in our previous research, with overlapping samples (Silton et al., 2010; Spielberg et al., 2015). A total of 256 trials were presented in 16 blocks (four congruent, four incongruent, and eight neutral) with intertrial intervals of $2000 \pm 225 \mathrm{~ms}$ (onset to onset). Neutral trials were intermixed (50\%) in congruent and incongruent blocks to prevent word reading strategies. A trial consisted of one word presented in one of four ink colors (red, yellow, green, or blue). Congruent (CON) trials were characterized by word meaning matching ink color (e.g., "GREEN" with green ink), whereas incongruent (INC) trials were characterized by word meaning differing from ink color (e.g., "GREEN" in red ink). In neutral (NEU) trials, word meaning and colors were unrelated (e.g., "CENTURY" in green ink). Control of stimulus order effects was achieved by each participant receiving one of eight stimulus orders. Participants were asked to respond via button press to the color of the word while ignoring the meaning of the word.

Data acquisition and preprocessing. Data acquisition was performed while participants were seated in a comfortable chair in a quiet room (for details, see Silton et al., 2010). EEG was recorded with a custom Falk Minow 64-channel cap with equidistant $\mathrm{Ag} / \mathrm{AgCl}$ electrodes. Electrode digitization followed cap placement for source localization purposes. The left mastoid served as the online reference for all sites. Impedances were below $20 \mathrm{k} \Omega$, which is appropriate given the high input impedance of the amplifier (James Long). Half-power amplifier band pass was 0.1$100 \mathrm{~Hz}$ with a sampling rate of $250 \mathrm{~Hz}$. Data analyses were performed with the MATLAB (The MathWorks) FieldTrip toolbox (Oostenveld et al., 2011) and the scikit-learn toolbox (Pedregosa et al., 2011) complemented by custom-written software. Trials with saccades or muscle artifact were excluded from analysis. On average $30.8 \pm 1.7 \mathrm{CON}$ trials and $28.3 \pm 3.2$ INC trials per subject entered the analysis. An independent components analysis (ICA) (Jung et al., 2001) was applied after demeaning and removing the linear trend from the data. Components associated with eye blink and cardiac activity were removed using ICA.

Spectral analysis. Spectral analysis was computed for each trial using a fast Fourier transformation with a $500 \mathrm{~ms}$ sliding window multiplied by a Hanning taper. The time window advanced in $50 \mathrm{~ms}$ increments and spectral resolution was $2 \mathrm{~Hz}$. Spectral power was calculated for each trial and then averaged across trials. Average baseline from -500 to $-250 \mathrm{~ms}$ before stimulus onset was subtracted and the data converted to units of decibels change from prestimulus baseline.

Source analysis. Anatomical MRI and EEG data were coregistered to a common coordinate system (Montreal Neurological Institute, MNI) by applying an initial coarse registration between the fiducial locations (nasion, left and right pre-auricular points) and subsequent refined matching between the scalp surface extracted from the MRI and digitized electrode positions. A three-compartment (skin, skull, and brain) pseudorealistic boundary element forward solution was constructed on the basis of a standard MRI (MNI152 linear template). Scalp EEG time series were projected into brain source space by applying a time-domain spatial filtering algorithm (linearly constrained minimum variance; Van Veen et al., 1997). This algorithm uses the covariance matrix of the EEG data to construct a spatial filter for a given location (voxel). These spatial filters were estimated for each participant on the basis of all trials and for the $1000 \mathrm{~ms}$ interval after stimulus onset. Subsequently, these filters were applied to the data to estimate the time series for each of the 11 network node locations previously reported as a network exhibiting greater hemodynamic coupling during high demands for inhibitory control (Spielberg et al., 2015). The other 237 participants completed the same protocol in the same laboratory at another time, providing a large repli- 
cation sample. MNI coordinates ( $x y z)$ and labels for these locations were as follows: right posterior middle frontal gyrus (MFG)/precentral, (44 10 40); right anterior insula, (38 19 6); right IFS, (47 35 20); right posterior middle temporal gyrus/superior temporal gyrus, (57 - 24-3); left posterior MFG/precentral, ( -48837 ); left inferior frontal gyrus (IFG) pars opercularis, (-52 21 18); right IFG pars opercularis, (53 14 20); medial precuneus, $(1-6155)$; right amygdala/hippocampus, $(23-10-15)$; left posterior superior frontal gyrus (SFG), (-17 14 65); and medial dACC, (0 26 34).

GC analysis. Spectrally resolved GC analysis (Granger, 1969; Ding et al., 2006) was used to identify potential directionality in internodal communication. Briefly, GC analyses represent the result of a comparison between two models: a univariate autoregressive model, in which the future behavior of time series $x$ is predicted from past values of time series $x$, and a bivariate autoregressive model, in which the prospective values of $x$ are predicted from the past values of time series $x$ and another time series $y$. Initially formulated in the time domain, GC can also be estimated in the frequency domain (Kamiński et al., 2001; Chen et al., 2006; Bastos and Schoffelen, 2015; for detailed review, see Bastos and Schoffelen, 2015), which requires the estimation of the spectral transfer matrix between a set of signals. The total interdependence $f_{\mathrm{x}, \mathrm{y}}(f)$ between two signals $x$ and $y$ is computed by their power spectral functions $S_{\mathrm{xx}}(f)$ and $S_{\mathrm{yy}}(f)$ and the corresponding crossspectral function $S_{\mathrm{xx}}(f)$ as follows:

$$
f_{x, y}(f)=\ln \left(\frac{S_{x x}(f) S_{y y}(f)}{|S(f)|}\right)
$$

The natural logarithm is denoted by $\ln$ and $S(f)=S_{\mathrm{xx}}(f) S_{\mathrm{yy}}(f)-S_{\mathrm{xy}}(f) S_{\mathrm{yx}}(f)$ using the coherence $C_{\mathrm{xy}}(f)$ between the two signals defined as follows:

$$
C_{x y}(f)=\frac{\left|S_{x y}(f)\right|^{2}}{\left(S_{x x}(f) S_{y y}(f)\right)}
$$

the total interdependence can be expressed as follows:

$$
f_{x, y}(f)=-\ln \left(1-C_{x y}(f)\right)
$$

Subsequently, the total interdependence can be decomposed into three causality terms as follows:

$$
f_{x, y}(f)=f_{x \rightarrow y}+f_{y \rightarrow x}+f_{x \cdot y}
$$

The first two terms denote the directed influence (over time) from $x$ to $y$ and $y$ to $x$, respectively, and the third term denotes the instantaneous dependence. The latter is often a consequence of, for example, common input from a third signal, nonlinear interactions between $x$ and $y$, and shared noise.

The time series were resegmented to include 500-1500 ms after stimulus onset to circumvent the presence of transient evoked activity at stimulus onset. For each trial, the Fourier coefficients were computed for the entire spectrum up to the Nyquist frequency after padding the data with zeros for $3 \mathrm{~s}$ at the beginning and the end of each trial to provide improved frequency resolution. A nonparametric matrix factorization of the crossspectral density matrix (Wilson, 1972; Dhamala et al., 2008) was applied to estimate the spectral transfer matrix.

It has been demonstrated that:

$$
f_{x \rightarrow y}(f)=-\ln \left(\frac{S_{x x}(f)}{\tilde{H}_{x x} \Sigma_{2} \tilde{H}_{x x}^{*}}\right)
$$

and

$$
f_{y \rightarrow x}(f)=-\ln \left(\frac{S_{y y}(f)}{\tilde{H}_{y y} \Gamma_{2} \tilde{H}_{y y}^{*}}\right)
$$

where $\Sigma_{2}$ and $\Gamma_{2}$ are the variance of the noise term in the bivariate model of signal $x$ and $y, \tilde{H}_{x x}$ is the spectral transfer function of the signal $x$, and * is the conjugate (Ding et al., 2006). Therefore, the instantaneous causality is represented by the difference in total interdependence of $x$ and $y$ and the sum of the causality terms $f_{\mathrm{x} \rightarrow y}$ and $f_{\mathrm{y} \rightarrow x}$. For a given pair of locations, GC was computed conditional on the presence of the remain- ing locations similar to the procedures applied previously (Wen et al., 2013; Bastos et al., 2015). Significant differences in GC between locations were determined on the basis of nonparametric cluster permutation tests (see next section). In addition, GC influences were computed twice: on the original and on the time-reversed time series. The latter strategy, proposed previously (Haufe et al., 2013; Winkler et al., 2015), accounts for the presence of so-called weak asymmetries as a possible interpretational confound, contributing to an apparent dominant directional drive between two areas. Briefly, as opposed to strong asymmetries, which are caused by actual time-lagged relations between signals, weak asymmetries are the consequence of differences in univariate signal properties (e.g., the local signal-to-noise ratio). Time reversal of the signals does not affect these univariate signal properties, whereas it should reverse the dominant direction of interaction. In other words, if the direction of the GC asymmetry is unchanged after the time reversal, then the GC is most likely artificial, whereas a reversal of the direction of the asymmetry implies a true time-lagged, and thus directed, relationship. Original versus time-reversed GC analysis outputs were compared statistically using the nonparametric permutation technique described above.

Statistical analysis. Statistical evaluation of the neuronal data from the sample described above was performed by a cluster-based approach based on permutations (Maris and Oostenveld, 2007). This approach identifies clusters of activity on the basis of whether the null hypothesis can be rejected while controlling for multiple comparisons. The test statistic for comparing condition A and condition B was built on the basis of relative changes defined as follows: test statistic $=[A-B] /[(A+B)]$. Behavioral performance $(\mathrm{RT})$ was expressed as a difference score, $\Delta \mathrm{RT}=$ $\mathrm{RT}_{\mathrm{IC}}-\mathrm{RT}_{\mathrm{C}}$. Relationships between neuronal and behavioral data were assessed by means of Spearman's rank-order correlations $(\rho)$. Effect sizes and exact $p$-values are reported when appropriate (i.e., parametric statistical tests).

Classification analysis. Classification with random forest models (Breiman, 2001) was used to distinguish the predictive strength of different variables, such as network characteristics and RT, on inhibitory control demands.

The random forest approach is able to handle highly correlated features notably well (Cutler et al., 2009). This specialty makes random forests well suited for the analysis of MEEG data, in which highly correlated variables are an often encountered problem (Lehmann et al., 2007; Donos et al., 2015; Westner et al., 2018). Furthermore, it provides a ranking of the informational value that each predictor had for the model (Breiman et al., 1984; Cutler et al., 2009), thereby enabling the direct comparison of predictor variables.

The random forest algorithm is an ensemble method aggregating the predictions of a large number of decision-tree classifiers (Breiman et al., 1984). Each decision tree partitions the data by adopting binary splits, aiming to reduce the impurity in the resulting subsets; that is, as many observations of a class as possible should be concentrated in one subset. To yield stable results even with outliers or highly correlated predictor variables, the random forest model introduces randomness at two levels. First, each tree in the ensemble is fit on a bootstrap sample of the data. Second, at each split within a tree, only a random subset of all predictors is searched to find the best predictor and split point to minimize the impurity in the resulting subsets. The high variance among a large number of such classification trees converges to a robust prediction when aggregating them across the ensemble.

Random forest classification was done using the scikit-learn toolbox (Pedregosa et al., 2011) in Python. Every model incorporated 5000 trees and considered $\sqrt{N_{\text {predictors }}}$ randomly drawn predictors at each split. Classifier performance was evaluated by using prediction accuracy within a fivefold cross-validation framework. Cross-validation prevents biased accuracy estimates because the model is always tested on data it was not trained on. Accuracy estimates were tested against chance level using a binomial test (Combrisson and Jerbi, 2015). To obtain a measure of the relative importance of each variable within the classification model, the Gini index was computed (Breiman et al., 1984; Cutler et al., 2009). This measure is based on the impurity reduction generated across all trees by a predictor and reflects the importance of this variable for the classifier's prediction. 
fMRI

A

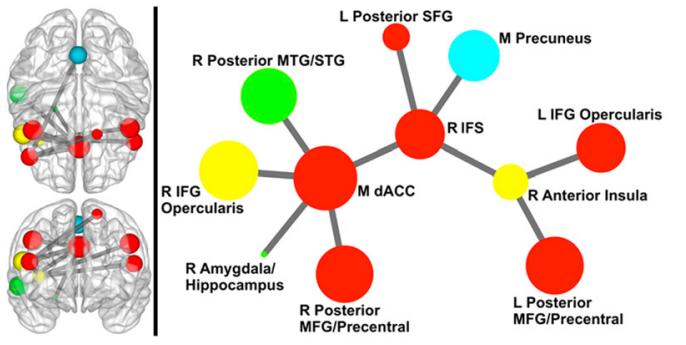

B

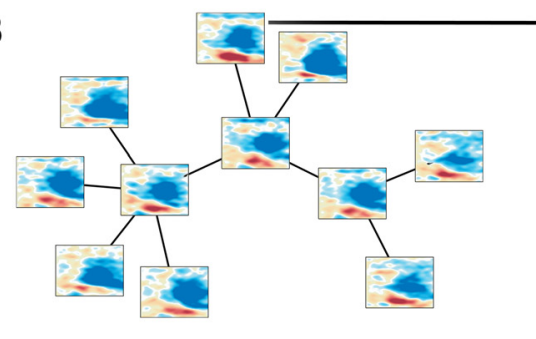

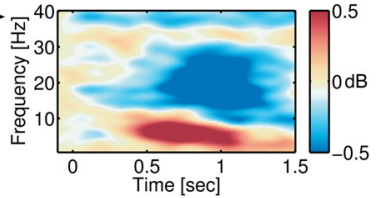

Figure 1. Neuronal dynamics in the hemodynamically informed cerebral network of inhibitory control $(n=333)$. $\boldsymbol{A}$, The network reported to exhibit greater hemodynamic coupling with increase in inhibitory control demands (reprinted with permission from Spielberg et al., 2015). Circle color represents module membership; circle size reflects node strength. $\boldsymbol{B}$, Time-frequency representation of power at locations corresponding to the topology in $\boldsymbol{A}$. Inset at right enlarges the time-frequency plot for a single exemplar node. Word onset was at $0 \mathrm{~ms}$. Warm and cold colors reflect power changes from prestimulus baseline expressed in decibels.

A
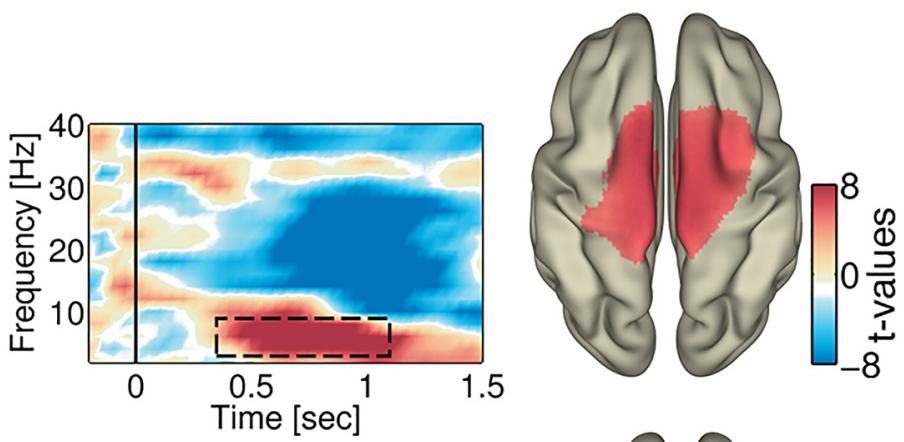

B

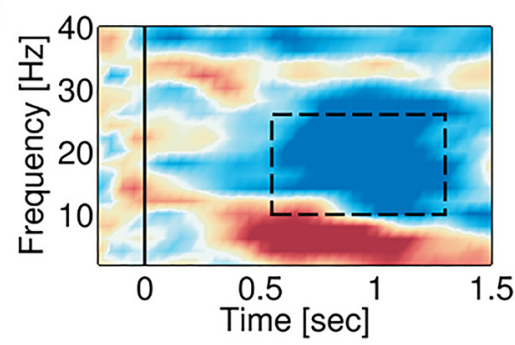

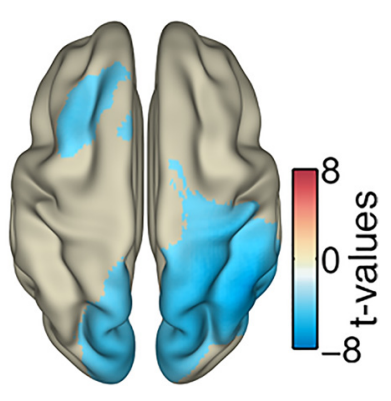

Figure 2. Whole-brain distribution of condition differences in theta and beta power $(n=333)$. $\boldsymbol{A}$, Left, Time-frequency representation of power for a representative node (SFG). Right, Difference in source-reconstructed theta power between INC and CON conditions for the time and frequency tile highlighted by the dashed rectangle in $\boldsymbol{A}$. $\boldsymbol{B}$, Left, Same as in $\boldsymbol{A}$, with the rectangle highlighting a different time-frequency combination. Right, Differences in source-reconstructed beta power between the INC and CON conditions for the time and frequency tile highlighted by the dashed rectangle in $A$. Warm and cold colors (thresholded at $p<$ 0.05 , corrected in right panels) reflect the range of $t$-values in both $\boldsymbol{A}$ and $\boldsymbol{B}$.

The first classification model was fit to predict congruent and incongruent class membership using theta-power values at network nodes as predictors. For each participant, baseline-adjusted theta power (averaged over $4-8 \mathrm{~Hz}$ and $500-800 \mathrm{~ms}$ ) was averaged within conditions and these averages were provided to the classifier, yielding 666 observations in total (333 participants $\times 2$ conditions).

In addition to the theta-power model, random forest classifiers were computed using baseline-adjusted beta power (averaged over 15-25 Hz and $800-1200 \mathrm{~ms}$ ), RT, and all possible combinations of these entities as predictors.

This approach was chosen over linear correlation analyses to compare different models (theta, beta, beta + RT, etc.). These comparisons are difficult to achieve via pure correlations between predictors. Moreover, linear models are not suitable for the aforementioned comparison because of the highly correlated nature of the predictors of interest.

\section{Results}

As expected, RT was longer on INC trials, with higher demand for inhibitory control, than on CON and NEU trials $(\mathrm{M} / \mathrm{SD}$ INC $=$

831.5/146.5, $\mathrm{CON}=636.8 / 103.2, \mathrm{NEU}=$ 645.2/92.5; $F_{(2,996)}=297.7, p<0.001$, Huynh-Feldt $\varepsilon=0.7$; INC vs NEU $t_{(332)}=33.1, p<0.001$, Cohen's $d=|1.52|$; INC vs CON $t_{(332)}=32.3, p<0.001$, Cohen's $d=|1.53|$; CON vs NEU $t_{(332)}=-2.9, p<$ 0.003 , Cohen's $d=|0.086|)$.

\section{Global network configuration of inhibitory control is dominated by} theta and alpha/beta neural oscillations Using the same participants $(n=96)$ and a replication sample of 237 participants (see Materials and Methods) to evaluate the oscillatory dynamics in the cerebral subnetwork (Fig. 1A) identified via fMRI (Spielberg et al., 2015), EEG data (same task recorded in a separate session; see Silton et al., 2010) were back-projected to the brain locations identified in that network. Time-frequency analysis of each EEG source revealed reliable modulations of theta $(4-7 \mathrm{~Hz})$ and alpha/beta $(10-30$ $\mathrm{Hz}$ ) oscillations (Fig. $1 B$ ), which is consistent with previous reports (Hanslmayr et al., 2008; Cavanagh and Frank, 2014; Cohen, 2014; Popov et al., 2015). Theta activity was higher in the INC condition than in the CON condition, starting 300 to $400 \mathrm{~ms}$ after word onset and lasting several hundred milliseconds. This increase was accompanied by a condition-specific (INC > CON) decrease in alpha/beta activity starting around $500 \mathrm{~ms}$ after word onset and lasting for $\sim 1000 \mathrm{~ms}$. Whole-brain source analysis revealed that theta generators were predominantly confined to frontal regions (Fig. $2 A$ ), whereas beta activity was most pronounced in parietal areas (Fig. 2B).

Inhibitory demands drive theta and alpha/beta oscillations Initial contrasts were performed with the $n=96$ sample reported previously (Spielberg et al., 2015) and shown in the top row of Figure 3. Figure $3 A$ illustrates significant (INC $-\mathrm{CON}$ ) condition differences in alpha/beta power $(p<0.01$, nonparametric cluster permutation approach, see Materials and Methods). As predicted, higher demand for inhibitory control was associated with an alpha/beta power decrease from prestimulus baseline. This decrease was inversely related to behavioral performance (Fig. 3B): slower responses were related to greater decrease in 
alpha/beta power (Fig. 2C). The $n=237$ sample replicated these effects (bottom row of Fig. 3). This much larger sample also showed more theta power in the highdemand INC condition $(p<0.01)$ (this effect was evident in the first sample but did not reach significance).

In summary, high demand for inhibitory control was related to reliable changes in theta and alpha/beta power. The latter in turn related to slower responses. The general consistency across the panels of Figure $1 B$ suggests that power changes were distributed throughout the entire network, precluding inferences about internodal communication from these initial analyses.

\section{SFG and dACC are primary nodes of prefrontal inhibitory control}

Internodal communication was assessed in the combined sample via spectrally resolved GC analysis (see Materials and Methods). Conditional GC was computed on original and reversed time series (Chen et al., 2006; Wen et al., 2013). Robust connectivity relationships are illustrated in Figure $4 A$. There were directionally asymmetric connections, especially in the theta range between SFG and dACC and between SFG and IFG, revealing a strong top-down influence of SFG over both dACC and IFG (GC spectra, $p<0.001$; Fig. $4 B$ ). Moreover, there was an exchange between frontal and parietal brain areas from precuneus to IFG and MFG in low theta-alpha frequency range $(7-10 \mathrm{~Hz} ; p<0.01$, nonparametric cluster permutation; Fig. $4 B$, middle). Finally, dACC exerted control over MFG at a range of frequencies, although this was particularly pronounced in the theta range $(p<0.001$; Fig. $4 B$, right $)$. These corticocortical relationships were reversed after time reversal of the original time series (Fig. 4C), which confirms that the reported lagged relationships between any two brain regions likely reflect unbiased directed effective influence of A on B beyond mere correlation. In summary, there was a dynamic exchange within frontal and between frontal and parietal brain areas: prefrontal control manifested in theta and parietal to frontal communication evident at higher frequencies overlapping with common posterior alpha oscillations $(8-13 \mathrm{~Hz})$.

\section{Inhibitory control is best predicted by combination of brain and behavioral variables}

The analyses presented above indicate that theta and beta oscillations reliably differed in high- and low-demand conditions and revealed effective connectivity patterns within the inhibitory control network. These results raise the question of the extent to which theta and beta power dynamics reflect high versus low demands for inhibitory control and perhaps mediate effects of these demands on overt performance.

The random forest machine learning algorithm was used to evaluate the predictive strength of theta, beta, and behavioral performance and their combinations for predicting the condition membership (INC or CON) of averaged data at the single-subject level. Random forest is an ensemble technique that aggregates the results of many decision trees into one classifier. These trees partition the data by implementing binary splits (Breiman, 2001). Every decision tree is built on random samples of observations and predictors. This ensemble approach makes random forest a
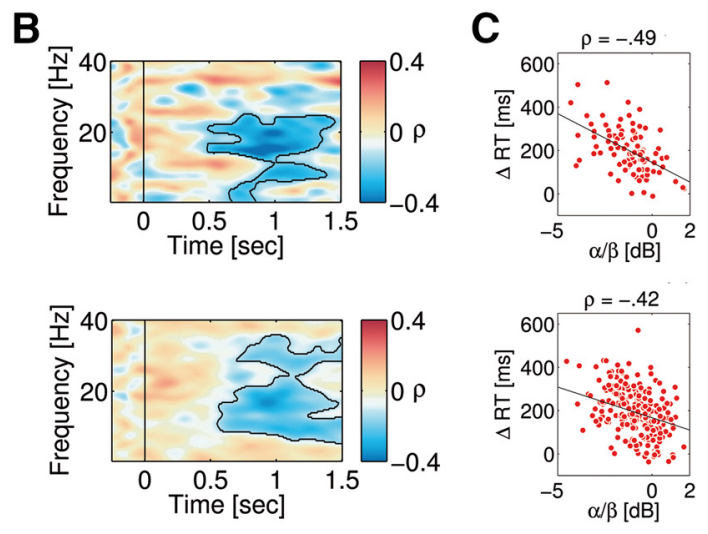

Figure 3. Task demand modulation of theta and alpha/beta power (top, $n=96$; bottom, $n=237$ ). $\boldsymbol{A}$, Time-frequency representation of power for a representative node (SFG). Color scale indicates condition difference (INC - CON) as change from scale indicates Spearman correlation. Highlighted areas indicate time-frequency tiles allowing the rejection of the null hypothesis. C, Scatterplots illustrating each participant's mean value over the respective outlined areas in $B$ and $\Delta R T$.

robust technique able to handle data with highly correlated predictors particularly well. Figure $5 A$ provides the classification results. Condition could be predicted above chance on the basis of any subset of the three variables, with all three in combination achieving $77 \%$ accuracy. Figure $1 B$ shows that both theta and beta oscillatory activity were distributed across the entire network, with some variability in amplitude. The information value of a particular node for prediction of inhibitory control demands was estimated by random forest models (Fig. 4B). Estimates of theta oscillatory activity derived from SFG were most important for accurate classification of condition membership (Fig. 5B, left). In the beta power model, parietal oscillations (precuneus; Fig. $5 B$, right) were the most important.

Figure $5 \mathrm{~A}$ suggested an interesting pattern: although theta by itself provided the lowest accuracy, the combination of theta and RT had higher classification accuracy than the combination of beta and RT. This suggests more variance shared by beta and RT. This post hoc hypothesis was addressed by first extracting the envelope of beta and theta activity as a function of time for each participant. These individual time courses were sorted according to mean RT across trials of each participant. The shift from beta decrease to increase coincident with button press in Figure $5 C$ suggests a close coupling of beta activity immediately preceding button press, whereas the time courses of theta and button press were more loosely associated, consistent with more variance shared by beta activity and RT.

In summary, the application of predictive analytics to neuroimaging data revealed the following: (1) neither behavioral nor brain-derived variables, but rather, a combination of both is the best predictor of cognitive state; (2) SFG and not dACC theta provides numerically higher feature importance during classification of low versus high demand for inhibitory control; and (3) posterior beta has the highest classification value and reliably tracks the onset of a behavioral outcome (RT).

\section{Discussion}

The present study evaluated the effective connectivity within a previously reported (Spielberg et al., 2015) network of inhibitory control during a color-word Stroop task. In a sample of 333 human volunteers, network nodes exhibiting greater interconnectivity during periods of higher demands for inhibitory control (Spielberg et al., 2015) are dominated by oscillatory activity in the 


\section{A}

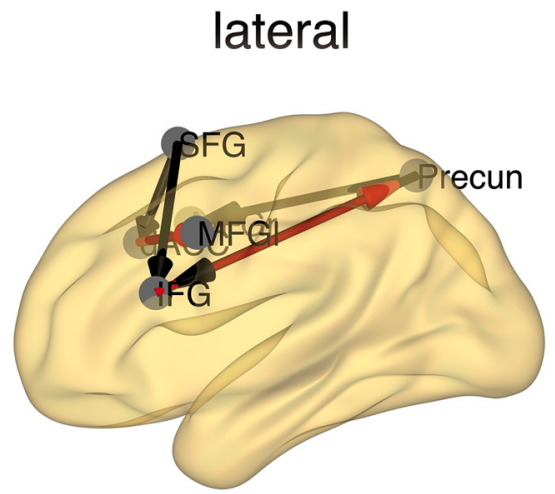

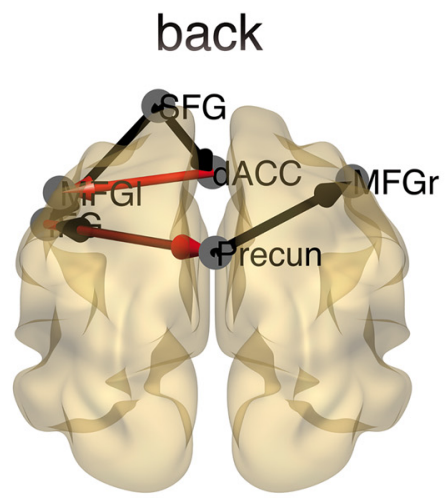

front

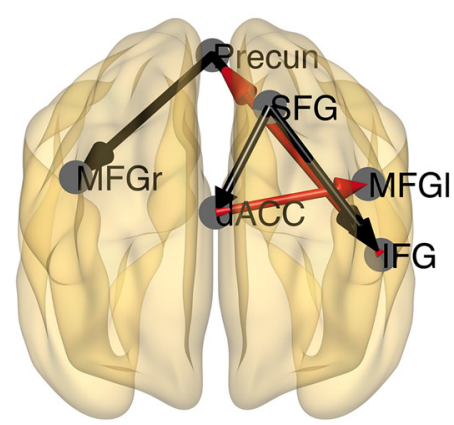

B
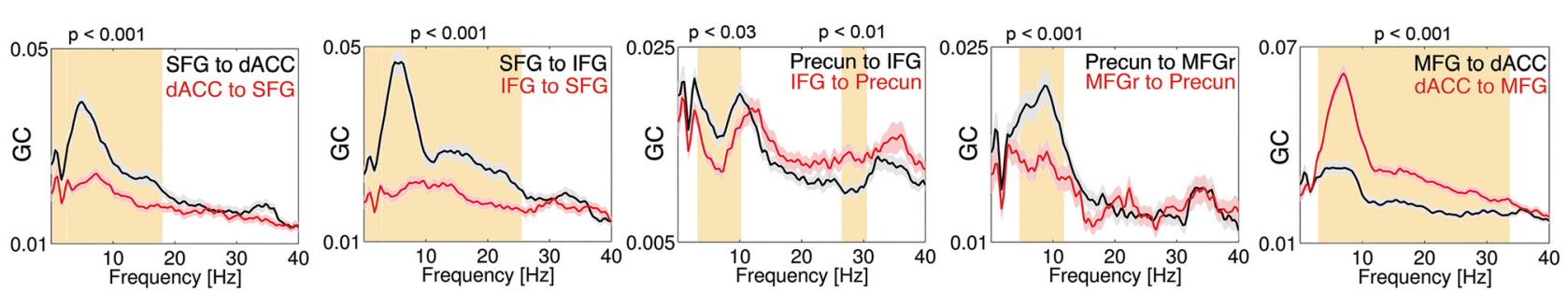

C
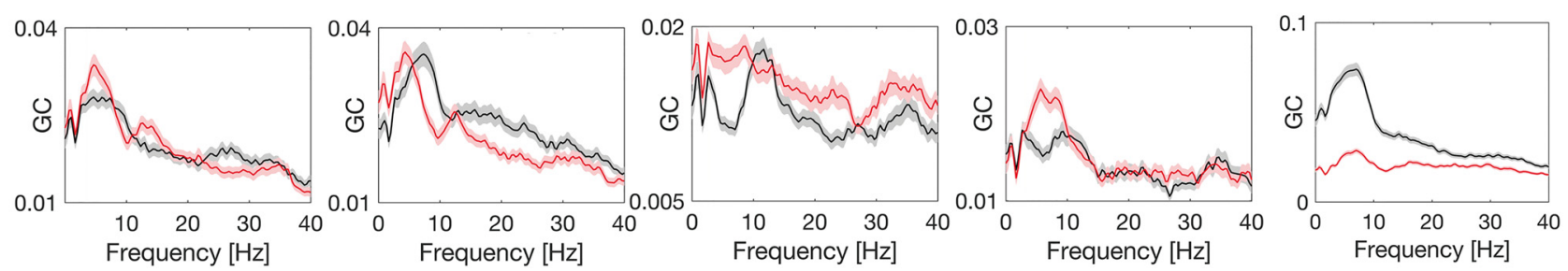

Figure 4. Effective connectivity patterns in the inhibitory control network ( $n=333$ ). $\boldsymbol{A}, \mathrm{GC}$ effects are illustrated in the brain models with arrows indicating the directionality of the respective connection. Arrows use the color scheme of the $\mathrm{GC}$ spectra illustrated in $\boldsymbol{B}$, where $\mathrm{GC}$ magnitude is depicted on the ordinate (log of ratio of $F$-values of the two models). Light-colored regions around spectra represent $1 \mathrm{SE}$. Higher values indicate stronger GC. Yellow shading indicates contiguous frequencies showing significant $\mathrm{GC}$ directionality differences. Bonferroni-corrected $p$-values ( $p<$ 0.01) are reported above the respective clusters. $\boldsymbol{C}$, Same as in $\boldsymbol{B}$ but for time-reversed time series (see Materials and Methods).

A

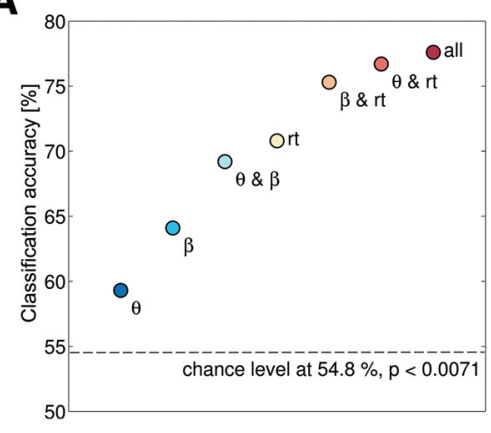

B

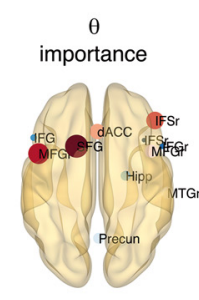

$\min$
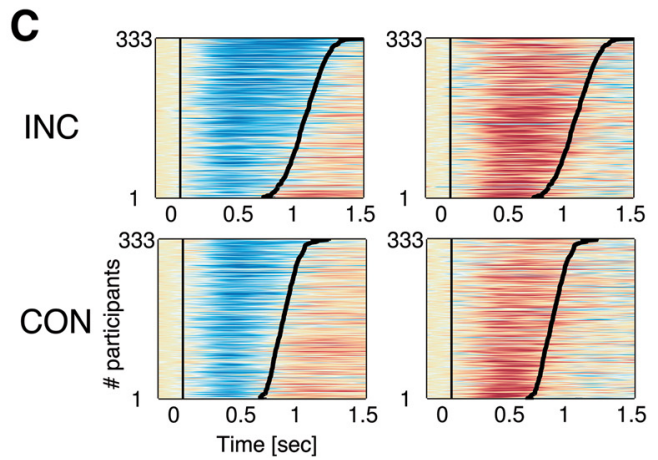

Figure 5. Classification performance of theta, beta, and RT ( $n=333$ ). $\boldsymbol{A}$, Classification accuracy differentiating condition membership (INC vs CON). $\boldsymbol{B}$, Brain models illustrating the importance of a given node for accurate classification of condition membership based on theta (left) and beta (right) activity. Importance is indicated by color and dot size. $C$, Time course of individual subjects' beta (left) and theta (right) activity change from prestimulus baseline. Each row represents a single participant, with participants sorted according to mean RT across trials (indicated by the black sigmoids). Color denotes amplitude change from baseline, with cold colors indicating decrease and hot colors indicating increase.

beta- and theta-frequency bands (Fig. 1). A broad frequency range around beta was related to RT (Fig. 3), whereas internodal effective connectivity was most pronounced around theta (Fig. 4), although the full dissociation was not tested. Participant brain state during conditions with high versus low demand for inhibitory control can be predicted by incorporating knowledge of both brain and behavior better than either alone (Fig. 5). Frontal (SFG) and parietal (precuneus) nodes were found to be most informative for inferences about inhibitory control, underscoring the functional specificity of network nodes in time, frequency, and space.

Spectrotemporal dynamics of the inhibitory control network The present study used the color-word Stroop task to evaluate the temporal course of critical nodes associated with inhibitory control processes. In addition to recruiting top-down inhibitory 
control mechanisms, the task required motor output (button press) to respond to the color of the word while ignoring the meaning of the word. Therefore, sensorimotor regions (e.g., premotor, primary motor, supplementary motor, and somatosensory cortices) were presumably recruited after conflict resolution and response selection to implement a behavioral response. Based on a large body of evidence (Cavanagh and Frank, 2014), we hypothesized that inhibitory control demands would be reflected in an increase in theta $(4-8 \mathrm{~Hz})$ oscillatory activity (Chmielewski et al., 2016), whereas behavioral performance (RT) would be predominantly associated with power modulations in alpha/beta $(10-30 \mathrm{~Hz})$ activity (Engel and Fries, 2010).

Present analyses (Figs. 1,2) are consistent with the literature linking theta oscillatory activity to inhibitory control (Cohen et al., 2008; Cavanagh et al., 2009; Hanslmayr et al., 2012; Cohen and Donner, 2013; Pastötter et al., 2013; Cavanagh and Frank, 2014; Cohen, 2014; Oehrn et al., 2014; Zavala et al., 2014; Chmielewski et al., 2016) and beta activity to conflict resolution (Fan et al., 2007; Cohen et al., 2008; Engel and Fries, 2010; Brittain et al., 2012; Pastötter et al., 2013). Replicating previous findings, theta activity was greater during higher inhibitory demand. This activity was spatially distributed, involving both frontal and parietal brain regions and not confined to a particular node (e.g., dACC). Paralleling theta findings, power decrease in the alpha/ beta range was greater during higher inhibitory demand and had similar spatial distribution because both were reliably present in each of the nodes displayed in the network in Figure 1B. In addition, a larger condition difference in alpha/beta power decrease was correlated with a larger RT condition difference. Typically, power decreases in the alpha/beta range are considered reflective of efficient cognitive performance (Hanslmayr et al., 2012; Frey et al., 2015), in which "efficiency" is typically operationalized behaviorally by higher accuracy and/or response speed (i.e., shorter RT). Present results extend these findings by demonstrating that RT could be a potentially misleading parameter in the context of inhibitory control tasks. Rather than response speed, reduction of alpha/beta activity was a better index of task engagement and effort. Despite being characterized by a clear midfrontal (theta) and posterior (alpha/beta) topography, both rhythms were evident throughout the entire network. The present study aimed to identify whether these rhythms fostered effective internodal information exchange.

\section{Effective network communication: a mechanism of upregulation of the desired response}

A number of connections exhibited significant effective connectivity even after controlling for spurious connections (see Materials and Methods). Theta evidenced a directed relationship from SFG to dACC and IFG (Fig. 4), whereas left hemisphere MFG was under dACC control via theta. These findings provide a novel view of the role of AACC in inhibitory control: that theta carries the information fed forward from SFG to dACC, perhaps communicating information about the interference itself; for example, the mismatch between the task-relevant verbal content and the interfering color. dACC appears to signal the need for maintenance of verbal information to left MFG, fostering response accuracy at the expense of speed. It is worth noting that communication within the frontal portion of this inhibitory control network was dominated primarily by theta activity despite the presence of alpha/beta activity. A directed parietofrontal influence was evident from precuneus to the middle and inferior portions of the frontal gyrus at theta frequencies (Fig. $4 B$, middle).
A potential alternative explanation for the present corticocortical interactions could be prolonged motor inhibition. A control analysis subsampling trials in INC and CON conditions to eliminate the difference in RT yielded largely similar corticocortical interactions, evidence against that nonspecific explanation. The present results are consistent with a recent proposal based on animal and human evidence arguing that cerebral communication feedback operates at alpha/beta frequencies, whereas feedforward exchange is propagated via theta- and gamma-frequency ranges (Bastos et al., 2015). According to this view, sensory information regarding both task-relevant and interfering information is initially propagated from parietal to frontal cortex. Within the frontal module, information about the desired response is actively propagated within a loop comprising SFG-dACC and MFG. GC analyses were performed for the entire spectrum from 0 to the Nyquist frequency of $125 \mathrm{~Hz}$ and no effects were observed for frequencies above $40 \mathrm{~Hz}$.

At a behavioral level, this processing is achieved at the expense of RT, in support of theories highlighting the selection and upregulation of the desired response rather than mere suppression of interfering input (Munakata et al., 2011). The present findings in the alpha/beta range provide further evidence for this view. First, accurate yet slower responses during high inhibitory demand were associated with greater decrease of alpha/beta activity. Although not part of the model evaluated here, it is well documented that decreases in alpha/beta activity before motor output originate mainly from primary motor regions (Davis et al., 2012; Pape and Siegel, 2016). Second, this motor cortex beta modulation is prolonged (Fig. $5 C$ ), nicely tracking the behavioral outcome (RT) on a subject-by-subject basis. Therefore, in addition to theta-mediated selection within the frontal subcircuit, a further upregulation of the desired response is reflected in deliberate, accuracy-directed, motor activation manifested in alpha/beta activity.

\section{Prediction of inhibitory control demands based on neural oscillations}

A further focus of the present study was the predictability of inhibitory demand on the basis of neural activity. Such a research question is often hampered by low sample size, but the large sample size of the present study enabled state-of-the-art predictive analyses. Both theta and beta activity were informative enough to classify high versus low levels of inhibitory demand above chance (Fig. 5). Classification performance was lowest for theta followed by beta and reached almost the level of RT alone when the two bands were combined. However, although betabased classification was better than theta-based classification, the combination of beta and RT was less accurate than the combination of theta and RT (Fig. 5A). This frequency-dependent classification specificity is further supported by the fact that theta in SFG and alpha/beta in precuneus were the most important brain regions for accurate classification. Therefore, combining three (theta, beta, and RT) out of, in principle, an infinite number of psychological and biological variables that can be associated with a given individual allows for accurate prediction of need for inhibitory control in at least three out of four trial averages (Fig. $5 A)$.

\section{Conclusions}

In the present study, a large sample of healthy human participants facilitated identification of mechanisms within a recently identified brain network supporting inhibitory control. Combining two imaging modalities, fMRI and EEG, led to several con- 
clusions. Information flow during demand for inhibitory control is performed primarily by theta and alpha/beta oscillations. Theta activity establishes directed frontoparietal communication. Extending common views of the role of dACC as a key structure in inhibitory control, the present results indicate that dACC is not simply a control region; it exerts control, yet is itself subject to control within a prefrontal subcircuit connecting with SFG and directing information to MFG. Inhibitory control thus involves a dynamic routing process during which the desired response is upregulated via theta-mediated effective connectivity within key nodes of the PFC and beta-mediated motor preparation. The present findings provide mechanistic insights into the biological implementation of a psychological phenomenon.

\section{References}

Anderson KL, Rajagovindan R, Ghacibeh GA, Meador KJ, Ding M (2010) Theta oscillations mediate interaction between prefrontal cortex and medial temporal lobe in human memory. Cereb Cortex 20:1604-1612. CrossRef Medline

Babapoor-Farrokhran S, Vinck M, Womelsdorf T, Everling S (2017) Theta and beta synchrony coordinate frontal eye fields and anterior cingulate cortex during sensorimotor mapping. Nat Commun 8:13967. CrossRef Medline

Bastos AM, Schoffelen JM (2015) A tutorial review of functional connectivity analysis methods and their interpretational pitfalls. Front Syst Neurosci 9:175. CrossRef Medline

Bastos AM, Vezoli J, Bosman CA, Schoffelen JM, Oostenveld R, Dowdall JR, De Weerd P, Kennedy H, Fries P (2015) Visual areas exert feedforward and feedback influences through distinct frequency channels. Neuron 85:390-401. CrossRef Medline

Breiman L (2001) Random forests. Machine Learning 45:5-32.

Breiman L, Friedman J, Stone CJ, Olshen RA (1984) Classification and regression trees. London: Taylor and Francis.

Brittain JS, Watkins KE, Joundi RA, Ray NJ, Holland P, Green AL, Aziz TZ, Jenkinson N (2012) A role for the subthalamic nucleus in response inhibition during conflict. J Neurosci 32:13396-13401. CrossRef Medline

Cavanagh JF, Frank MJ (2014) Frontal theta as a mechanism for cognitive control. Trends Cogn Sci 18:414-421. CrossRef Medline

Cavanagh JF, Cohen MX, Allen JJ (2009) Prelude to and resolution of an error: EEG phase synchrony reveals cognitive control dynamics during action monitoring. J Neurosci 29:98-105. CrossRef Medline

Chen Y, Bressler SL, Ding M (2006) Frequency decomposition of conditional granger causality and application to multivariate neural field potential data. J Neurosci Methods 150:228-237. CrossRef Medline

Chmielewski WX, Mückschel M, Dippel G, Beste C (2016) Concurrent information affects response inhibition processes via the modulation of theta oscillations in cognitive control networks. Brain Struct Funct 221: 3949-3961. CrossRef Medline

Cohen MX (2014) A neural microcircuit for cognitive conflict detection and signaling. Trends Neurosci 37:480-490. CrossRef Medline

Cohen MX, Donner TH (2013) Midfrontal conflict-related theta-band power reflects neural oscillations that predict behavior. J Neurophysiol 110:2752-2763. CrossRef Medline

Cohen MX, Ridderinkhof KR, Haupt S, Elger CE, Fell J (2008) Medial frontal cortex and response conflict: evidence from human intracranial EEG and medial frontal cortex lesion. Brain Res 1238:127-142. CrossRef Medline

Cohen MX, Bour L, Mantione M, Figee M, Vink M, Tijssen MA, van Rootselaar AF, van den Munckhof P, Schuurman PR, Denys D (2012) Top-downdirected synchrony from medial frontal cortex to nucleus accumbens during reward anticipation. Hum Brain Mapp 33:246-252. CrossRef Medline

Combrisson E, Jerbi K (2015) Exceeding chance level by chance: the caveat of theoretical chance levels in brain signal classification and statistical assessment of decoding accuracy. J Neurosci Methods 250:126-136. CrossRef Medline

Cutler A, Cutler DR, Stevens JR (2009) Tree-based methods. In: Highdimensional data analysis in cancer research (Li X, ve Xu R, ed), pp 1-19. New York: Springer.

Davis NJ, Tomlinson SP, Morgan HM (2012) The role of beta-frequency neural oscillations in motor control. J Neurosci 32:403-404. CrossRef Medline
Dhamala M, Rangarajan G, Ding M (2008) Estimating granger causality from fourier and wavelet transforms of time series data. Phys Rev Lett 100:018701. CrossRef Medline

Ding M, Chen Y, Bressler S (2006) Granger causality: basic theory and application to neuroscience. In: Handbook of time series analysis (Schelter B, Winterhalder M, Timmer J, eds), pp 451-474. Weinheim, Germany: Wiley-VCH Verlage.

Donos C, Dümpelmann M, Schulze-Bonhage A (2015) Early seizure detection algorithm based on intracranial EEG and random forest classification. Int J Neural Syst 25:1550023. CrossRef Medline

Engel AK, Fries P (2010) Beta-band oscillations-signalling the status quo? Curr Opin Neurobiol 20:156-165. CrossRef Medline

Fan J, Byrne J, Worden MS, Guise KG, McCandliss BD, Fossella J, Posner MI (2007) The relation of brain oscillations to attentional networks. J Neurosci 27:6197-6206. CrossRef Medline

Frey JN, Ruhnau P, Weisz N (2015) Not so different after all: the same oscillatory processes support different types of attention. Brain Res 1626: 183-197. CrossRef Medline

Granger CWJ (1969) Investigating causal relations by econometric models and cross-spectral methods. Econometrica 37:424-438. CrossRef

Hanslmayr S, Pastötter B, Bäuml KH, Gruber S, Wimber M, Klimesch W (2008) The electrophysiological dynamics of interference during the stroop task. J Cogn Neurosci 20:215-225. CrossRef Medline

Hanslmayr S, Staudigl T, Fellner MC (2012) Oscillatory power decreases and long-term memory: the information via desynchronization hypothesis. Front Hum Neurosci 6:74. CrossRef Medline

Haufe S, Nikulin VV, Müller KR, Nolte G (2013) A critical assessment of connectivity measures for EEG data: a simulation study. Neuroimage 64:120-133. CrossRef Medline

Jung TP, Makeig S, McKeown MJ, Bell AJ, Lee TW, Sejnowski TJ (2001) Imaging brain dynamics using independent component analysis. Proceedings of the IEEE Institute of Electrical and Electronics Engineers 89: 1107-1122. CrossRef Medline

Kamiński M, Ding M, Truccolo WA, Bressler SL (2001) Evaluating causal relations in neural systems: granger causality, directed transfer function and statistical assessment of significance. Biol Cybern 85:145-157. CrossRef Medline

Lehmann C, Koenig T, Jelic V, Prichep L, John RE, Wahlund LO, Dodge Y, Dierks T (2007) Application and comparison of classification algorithms for recognition of Alzheimer's disease in electrical brain activity (EEG). J Neurosci Methods 161:342-350. CrossRef Medline

Maris E, Oostenveld R (2007) Nonparametric statistical testing of EEG-and MEG-data. J Neurosci Methods 164:177-190. CrossRef Medline

Michalareas G, Vezoli J, van Pelt S, Schoffelen JM, Kennedy H, Fries P (2016) Alpha-beta and gamma rhythms subserve feedback and feedforward influences among human visual cortical areas. Neuron 89:384-397. CrossRef Medline

Munakata Y, Herd SA, Chatham CH, Depue BE, Banich MT, O’Reilly RC (2011) A unified framework for inhibitory control. Trends Cogn Sci 15: 453-459. CrossRef Medline

Oehrn CR, Hanslmayr S, Fell J, Deuker L, Kremers NA, Do Lam AT, Elger CE, Axmacher N (2014) Neural communication patterns underlying conflict detection, resolution, and adaptation. J Neurosci 34:10438-10452. CrossRef Medline

Oldfield RC (1971) The assessment and analysis of handedness: the edinburgh inventory. Neuropsychologia 9:97-113. CrossRef Medline

Oostenveld R, Fries P, Maris E, Schoffelen JM (2011) FieldTrip: open source software for advanced analysis of MEG, EEG, and invasive electrophysiological data. Comput Intell Neurosci 2011:156869. CrossRef Medline

Pape AA, Siegel M (2016) Motor cortex activity predicts response alternation during sensorimotor decisions. Nat Commun 7:13098. CrossRef Medline

Pastötter B, Dreisbach G, Bäuml KH (2013) Dynamic adjustments of cognitive control: oscillatory correlates of the conflict adaptation effect. J Cogn Neurosci 25:2167-2178. CrossRef Medline

Pedregosa F, Varoquaux G, Gramfort A, Michel V, Thirion B, Grisel O, Blondel M, Prettenhofer P, Weiss R, Dubourg V, Vanderplas J, Passos A, Cournapeau D, Brucher M, Perrot M, Duchesnay E (2011) Scikit-learn: machine learning in Python. Journal of Machine Learning Research 12:28252830.

Popov T, Wienbruch C, Meissner S, Miller GA, Rockstroh B (2015) A mech- 
anism of deficient interregional neural communication in schizophrenia. Psychophysiology 52:648-656. CrossRef Medline

Popov T, Kastner S, Jensen O (2017) FEF-controlled alpha delay activity precedes stimulus-induced gamma-band activity in visual cortex. J Neurosci 37:4117-4127. CrossRef Medline

Richter CG, Thompson WH, Bosman CA, Fries P (2017) Top-down beta enhances bottom-up gamma. J Neurosci 37:6698-6711. CrossRef Medline

Schoffelen JM, Hultén A, Lam N, Marquand AF, Uddén J, Hagoort P (2017) Frequency-specific directed interactions in the human brain network for language. Proc Natl Acad Sci U S A 114:8083-8088. CrossRef Medline

Silton RL, Heller W, Towers DN, Engels AS, Spielberg JM, Edgar JC, Sass SM, Stewart JL, Sutton BP, Banich MT, Miller GA (2010) The time course of activity in dorsolateral prefrontal cortex and anterior cingulate cortex during top-down attentional control. Neuroimage 50:1292-1302. CrossRef Medline

Spielberg JM, Miller GA, Heller W, Banich MT (2015) Flexible brain network reconfiguration supporting inhibitory control. Proc Natl Acad Sci U S A 112:10020-10025. CrossRef Medline

Swann N, Tandon N, Canolty R, Ellmore TM, McEvoy LK, Dreyer S, DiSano M, Aron AR (2009) Intracranial EEG reveals a time- and frequencyspecific role for the right inferior frontal gyrus and primary motor cortex in stopping initiated responses. J Neurosci 29:12675-12685. CrossRef Medline

van Kerkoerle T, Self MW, Dagnino B, Gariel-Mathis MA, Poort J, van der Togt C, Roelfsema PR (2014) Alpha and gamma oscillations character- ize feedback and feedforward processing in monkey visual cortex. Proc Natl Acad Sci U S A 111:14332-14341. CrossRef Medline

Van Veen BD, van Drongelen W, Yuchtman M, Suzuki A (1997) Localization of brain electrical activity via linearly constrained minimum variance spatial filtering. IEEE Trans Biomed Eng 44:867-880. CrossRef Medline

Wang C, Rajagovindan R, Han SM, Ding M (2016) Top-down control of visual alpha oscillations: sources of control signals and their mechanisms of action. Front Hum Neurosci 10:15. CrossRef Medline

Wen X, Rangarajan G, Ding M (2013) Multivariate Granger causality: an estimation framework based on factorization of the spectral density matrix. Philos Trans A Math Phys Eng Sci 371:20110610. CrossRef Medline

Westner BU, Dalal SS, Hanslmayr S, Staudigl T (2018) Across-subjects classification of stimulus modality from human MEG high-frequency activity. PLoS Comput Biol 14:e1005938. CrossRef Medline

Wheaton L, Fridman E, Bohlhalter S, Vorbach S, Hallett M (2009) Left parietal activation related to planning, executing and suppressing praxis hand movements. Clin Neurophysiol 120:980-986. CrossRef Medline

Wilson G (1972) The factorization of matrical spectral densities. SIAM J Appl Math 23:420-426. CrossRef

Winkler I, Haufe S, Porbadnigk AK, Müller KR, Dähne S (2015) Identifying granger causal relationships between neural power dynamics and variables of interest. Neuroimage 111:489-504. CrossRef Medline

Zavala BA, Tan H, Little S, Ashkan K, Hariz M, Foltynie T, Zrinzo L, Zaghloul KA, Brown P (2014) Midline frontal cortex low-frequency activity drives subthalamic nucleus oscillations during conflict. J Neurosci 34: 7322-7333. CrossRef Medline 